\title{
CHAETOPHRACTUS TARIJENSIS (XENARTHRA, DASYPODIDAE) ¿UN ENDEMISMO DEL VALLE DE TARIJA (BOLIVIA)? IMPLICANCIAS PALEOBIOGEOGRÁFICAS
}

\author{
SANTIAGO RODRIGUEZ-BUALÓ \\ Centro de Ecología Aplicada del Litoral (CECOAL-CONICET) y Universidad Nacional del Nordeste, \\ Ruta 5, km 2,5 (3400), Corrientes, provincia de Corrientes, Argentina.santiago_mr@hotmail.com \\ ESTEBAN SOIBELZON, ALEJO CARLOS SCARANO \\ Departamento Científico de Paleontología de Vertebrados, Museo de La Plata, Paseo del Bosque s/nº, 1900, \\ La Plata, Argentina.esoibelzon@fcnym.unlp.edu.ar, scarano@cnym.unlp.edu.ar
}

ALFREDO EDUARDO ZURITA

Centro de Ecología Aplicada del Litoral (CECOAL-CONICET) y Universidad Nacional del Nordeste, Ruta 5, km 2,5 (3400), Corrientes, provincia de Corrientes, Argentina.aezurita74@yahoo.com.ar

\begin{abstract}
CHAETOPHRACTUS TARIJENSIS (XENARTHRA, DASYPODIDAE). AN ENDEMISM FROM THE TARIJA VALLEY (BOLIVIA)? PALEOBIOGEOGRAPHICAL IMPLICATIONS. Chaetophractus tarijensis (Ameghino) is a dasypodid restricted to the Pleistocene of the Tarija Valley (Bolivia). In this contribution we study the type specimen of $C$. tarijensis and referred specimens to this species, and establish comparisons with extant Dasypodidae Euphractinae. The analysis of the present study reveal that the characters used by Florentino Ameghino (e.g. the underdevelopment of the angular portion of the ramus of the mandible and the cylindrical shape of the last molariform) do not allow the differentiation with Chaetopractus (Desmarest). Also, a principal components analysis was performed with cranial measurements. All these evidence support the hypothesis that $C$. tarijensis is a synonym of $C$. villosus. From a paleobiogeographic point of view, these results are important because it indicates that $C$. tarijensis is not one of the few supposedly endemic species of the of the Tarija Valley.
\end{abstract}

Key words: Cingulata, Euphractinae, Pleistocene, Quaternary, South America, taxonomy.

RESUMO - Chaetophractus tarijensis (Ameghino) é um dasipodídeo restrito ao Pleistoceno do Vale de Tarija (Bolívia). A partir do estudo do exemplar tipo de C. tarijensis, dos materiais referidos a esta espécie e da comparação com outros Dasypodidae Euphractinae atuais, se conclui que os caracteres utilizados por Florentino Ameghino (e.g. o parco desenvolvimento da porção angular do ramo ascendente da mandíbula e a forma cilíndrica do último molariforme) não permitem diferenciá-la de Chaetopractus (Desmarest). Estas diferenças são também consistentes com os resultados obtidos por meio de uma análise de componentes principais, na qual $C$. tarijensis seria um sinônimo de C. villosus. Tais resultados são importantes do ponto de vista paleobiogeográfico, dado que seria outro caso de espécie originalmente proposta como endêmica do Vale de Tarija, porém que, segundo nossos recentes estudos, não seria válida.

Palavras-chave: Cingulata, Euphractinae, Pleistoceno, Quaternário, América do Sul, taxonomia.

\section{INTRODUCCIÓN}

Los Dasypodidae (Xenarthra, Cingulata) se encuentran exclusivamente en el continente Americano, en donde poseen una amplia distribución geográfica (entre los $40^{\circ} \mathrm{N} / 40^{\circ} \mathrm{S}$ ) y temporal (desde el Eoceno temprano hasta la actualidad; ver Carlini \& Scillato-Yané 1999; Ciancio \& Carlini, 2008; Krmpotic et al., 2009). Los representantes vivientes se agrupan en nueve géneros y 21 especies (Wetzel, 1985; Nowak, 1999; Wetzel et al., 2007). Asimismo, se conoce una mayor diversidad de fauna fósil, con aproximadamente 40 géneros y unas 200 especies extintas (Scillato-Yané, 1982; Mones, 1986; McKenna \& Bell, 1997).

De acuerdo con McKenna \& Bell (1997), los Dasypodidae están divididos en tres subfamilias: Dasypodinae, Euphractinae y Tolypeutinae, de las cuales las dos primeras incluyen la mayor parte de las especies, tanto extintas como actuales. El rasgo anatómico más evidente de la familia es la presencia de zonas acorazadas en distintas regiones del cuerpo (Krmpotic et al., 2009; Ciancio et al., 2012) y que están integradas por osteodermos coalescentes articulados. Ellos cubren el dorso de la cabeza (escudete cefálico) y del 


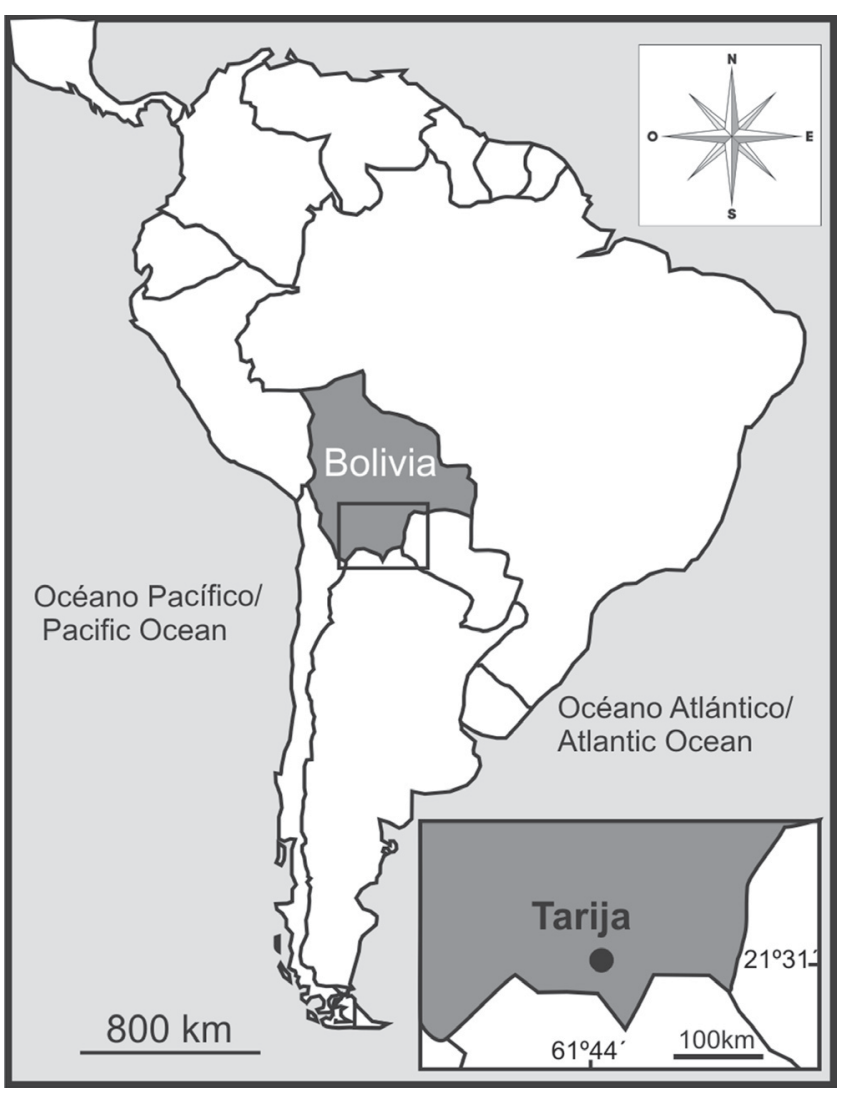

Figura 1. Mapa de ubicación, mostrando la localidad de donde provienen los restos de Chaetophractus tarijensis (Ameghino, 1902).

Figure 1. Location map showing the provenance of Chaetophractus tarijensis (Ameghino, 1902).

tronco (coraza dorsal), y envuelven la cola (estuche caudal) (excepto en Cabassous McMurtrie, 1931) (Soibelzon et al., 2012, fig. 1). Estos osteodermos pueden también estar presentes aislados en el tegumento del rostro, de la región ventral del tronco y dorsal de los miembros (Krmpotic et al., 2009).

El Valle de Tarija (Bolivia) es uno de los yacimientos pleistocenos más importantes de América del Sur y ha sido motivo de numerosas publicaciones científicas (ver comentario en Tonni et al., 2009 y la bibliografía allí citada). Se ubica a unos $2000 \mathrm{msnm}$ y posee una extensión geográfica de unos $4500 \mathrm{~km}^{2}$ (Figura 1). Desde una perspectiva geológica, los sedimentos fluviales que rellenan el Valle de Tarija corresponden a la Formación Tolomosa (ex Formación Tarija, ver Coltorti et al., 2010), la cual yace de manera discordante sobre un basamento Paleozoico (Silúrico-Devónico). La edad de la fauna de Tarija, en comparación con el esquema pampeano de Cione \& Tonni (2005), varía según los diferentes autores desde exclusivamente Ensenadense (ca. 2-0,5 Ma) (MacFadden et al., 1983; MacFadden \& Shokey, 1997; MacFadden, 2000; MacFadden et al., 2013) a exclusivamente Lujanense (ca. 40-10 Ka) (Coltorti et al., 2007, 2010). Sin embargo, Tonni et al. (2009) señalan que en comparación con el estándar pampeano, la fauna de Tarija no es exclusivamente Ensenadense ni Lujanense, dado que, si bien el grueso de la fauna es comparable a la del Lujanense pampeano, existen algunos taxones exclusivos del Ensenadense pampeano. Tonni et al. (2009, p. 63) proponen "the survival in this area of taxa recorded in the typical Ensenadan (i.e. G. munizi and A. angustidens) could be due to a local continued existence of these species", posiblemente debido a diferencias latitudinales.

Más allá de estas controversias, la fauna de Tarija es típicamente pleistocena, no obstante presenta diferencias con otras faunas sincrónicas de América del Sur. Estas diferencias corresponden tanto a una mayor frecuencia de algunos taxones (e.g. Tapirus Brünnich, 1772, Cuvieronius Osborn, 1923) como a la presencia de numerosas (ca. 10) especies supuestamente endémicas. En relación a esto último, estudios recientes indican la presencia de al menos cuatro de estos taxones endémicos en otras faunas de América del Sur [e.g. Scelidodon tarijensis (Gervais \& Ameghino, 1880), Arctotherium tarijense Ameghino, 1902] (Soibelzon et al., 2011). Asimismo, la paleofauna de Tarija requiere de modernas revisiones taxonómicas que ayuden a esclarecer la problemática tanto de la antigüedad, como de los endemismos. Este último es el caso de Chaetophractus tarijensis (Ameghino, 1902).

Entre los Euphractinae (Xenarthra, Dasypodidae) fósiles hallados en el Pleistoceno del Valle de Tarija, tradicionalmente se han citado a Euphractus cf. E. sexcinctus (Linnaeus, 1758), Chaetophractus cf. C. villosus (Desmarest, 1804) y C. tarijensis (Ameghino, 1902; Hoffstetter, 1963; Marshall \& Sempere, 1991; Tonni et al., 2009). Actualmente, las especies registradas son: E. sexcinctus, C. villosus, C. vellerosus (Gray, 1865) y C. nationi (Thomas, 1894).

En la presente contribución se discute la validez taxonómica de Chaetophractus tarijensis, y por lo tanto su carácter de taxón endémico de los depósitos cuaternarios del Valle de Tarija. Estudios paleofaunísticos y paleobiogeográficos realizados sobre la fauna de los Euphractinae (Dasypodidae) de esta localidad resultan relevantes a la hora de proponer esquemas biogeográficos y establecer correlaciones biocronológicas con otras áreas de América del Sur.

\section{MATERIAL Y MÉTODOS}

A fin de analizar la validez de Chaetophractus tarijensis se estudió el ejemplar Tipo (MACN 1612), un espécimen referido a esta especie (YPM PU 16612) (Figura 2) y otros ejemplares actuales de C. villosus $(\mathrm{n}=25), C$. vellerosus $(\mathrm{n}=32), C$. nationi $(\mathrm{n}=1)$ y Euphractus sexcinctus $(n=15)$, depositados en las colecciones del MACN, MLP y AAC. Se tomaron medidas equivalentes a las tomadas por Ameghino (1902) (LM, LP, DTD y DTI, AP-M3, LSDS, LSDM, ARH) y medidas nuevas propuestas en este trabajo (AP-M3, AP-M4, AC, PP, LSM, LS, ML) (Figura 3). Para ello se eligieron ejemplares que fuesen de igual estadio ontogenético que el ejemplar tipo (adultos), utilizándose como criterio para la determinación que las suturas entre el basioccipital y el basiesfenoides, y entre este último con el preesfenoides estuviesen soldadas. Cabe destacar que Morris 

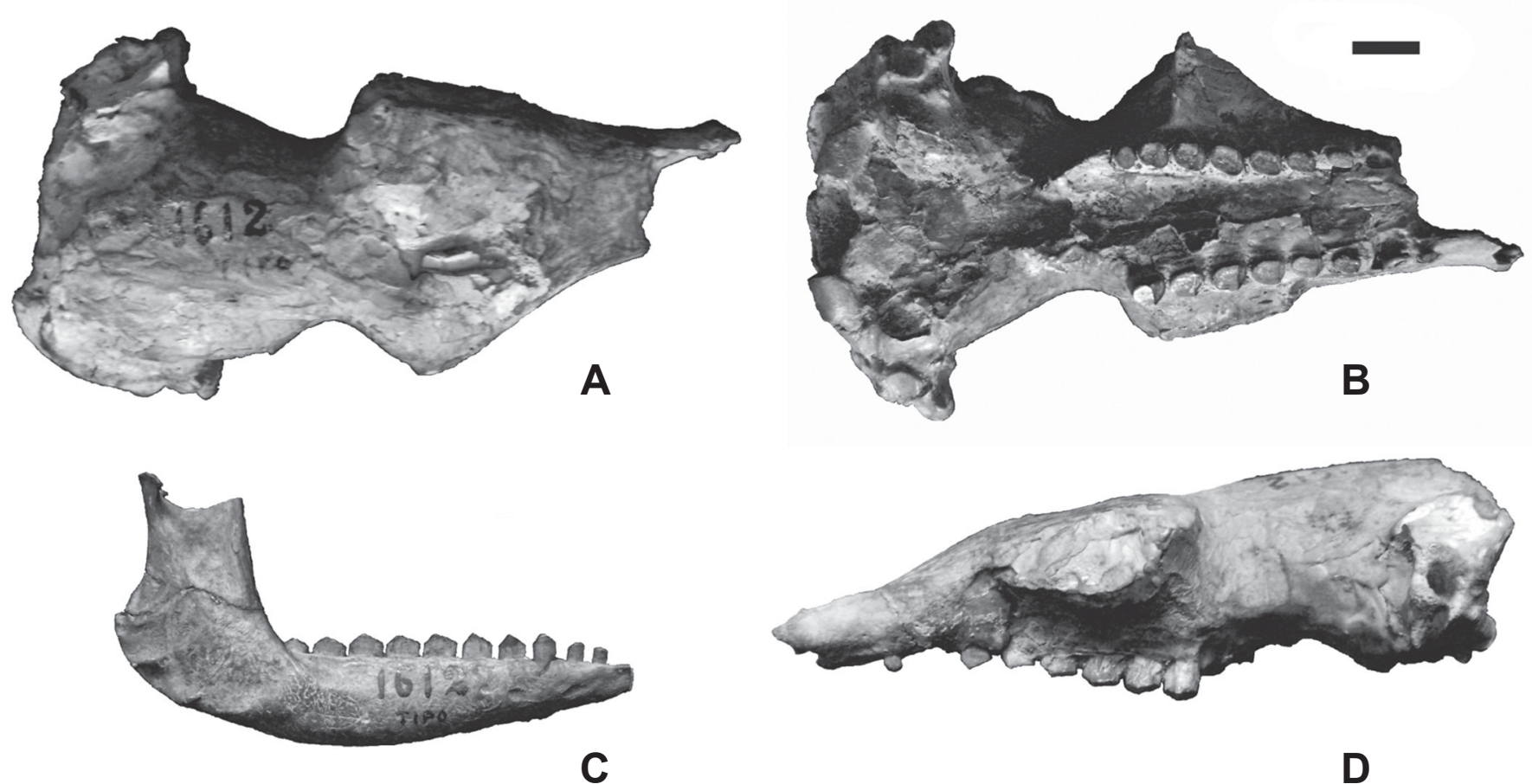

C
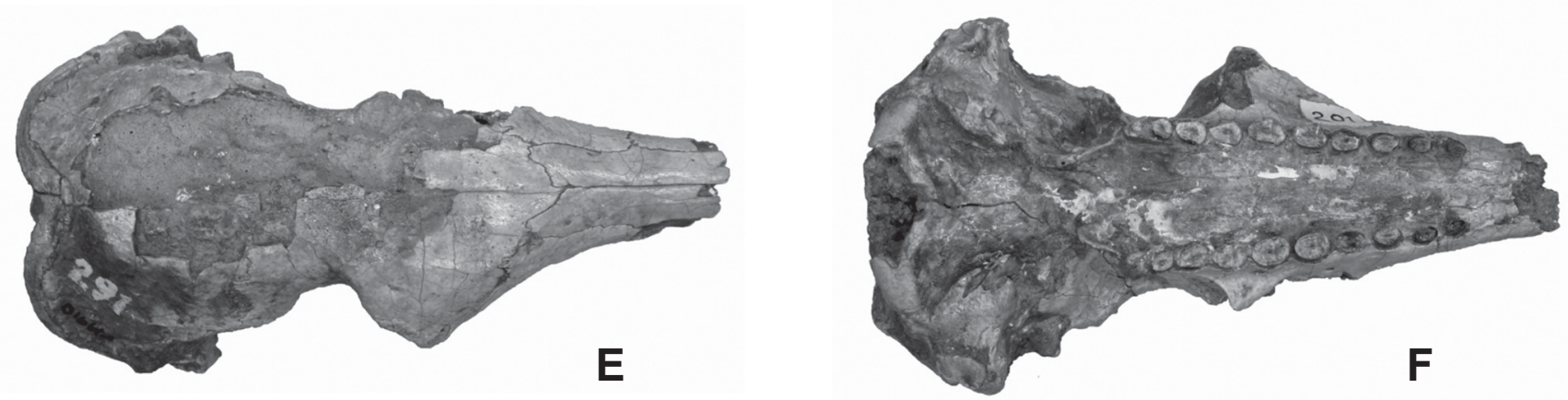

Figura 2. Chaetophractus tarijensis. A-D, ejemplar tipo (MACN 1612). Cráneo en vistas dorsal (A), ventral (B), lateral (D); C, hemimandíbula derecha. E-F, material referido (YMPMU 16612). Cráneo en vistas dorsal (E), ventral (F). Escala $=10 \mathrm{~mm}$.

Figure 2. Chaetophractus tarijensis. A-D, type material (MACN 1612). Skull dorsal (A), ventral (B), lateral (D) views; C, right hemimandible. E-F, referred material (YMPMU 16612). Skull in dorsal (E), ventral $(\mathbf{F})$ views. Scale bar $=10 \mathrm{~mm}$.

(1972) y Rager et al. (2013) indican que estos no son criterios completamente confiables para la determinación de la edad. Así, por ejemplo, el material de C. nationi (MACN 35308) no presenta la sutura entre el basiesfenoides y el preesfenoides soldada, pero si el resto de las suturas craneanas, por ello se lo incluye igualmente en el análisis.

Las medidas seleccionadas fueron las siguientes:

(i) longitud del cráneo desde la parte posterior de los cóndilos hasta la parte más anterior del pre-maxilar (LM).

(ii) longitud de la parte palatina del premaxilar delante del M1 (LP).

(iii) longitud de la sínfisis mandibular (LSM).

(iv) longitud de la porción sinfisiaria de la mandíbula delante del $\mathrm{m} 1$ (LS). (v) ancho del paladar a nivel del M3 (AP-M3), M4 (APM4) y M5 (AP-M5).

(vi) longitud de la serie dentaria superior (LSDS).

(vii) longitud serie dentaria inferior (LSDM).

(viii) alto de la rama horizontal de la mandíbula debajo del m8 (ARH).

(ix) longitud de la mandíbula: distancia extremo anterior de la sínfisis-borde posterior de la rama ascendente (ML).

(x) diámetro transverso máximo a nivel del meato auditorio derecho (DTD).

(xi) diámetro transverso máximo a nivel del meato auditorio izquierdo (DTI).

(xii) ancho del cráneo en vista dorsal a nivel del M3 (AC).

(xiii) distancia entre la parte posterior del M9 y la parte posterior de los cóndilos occipitales (PP). 

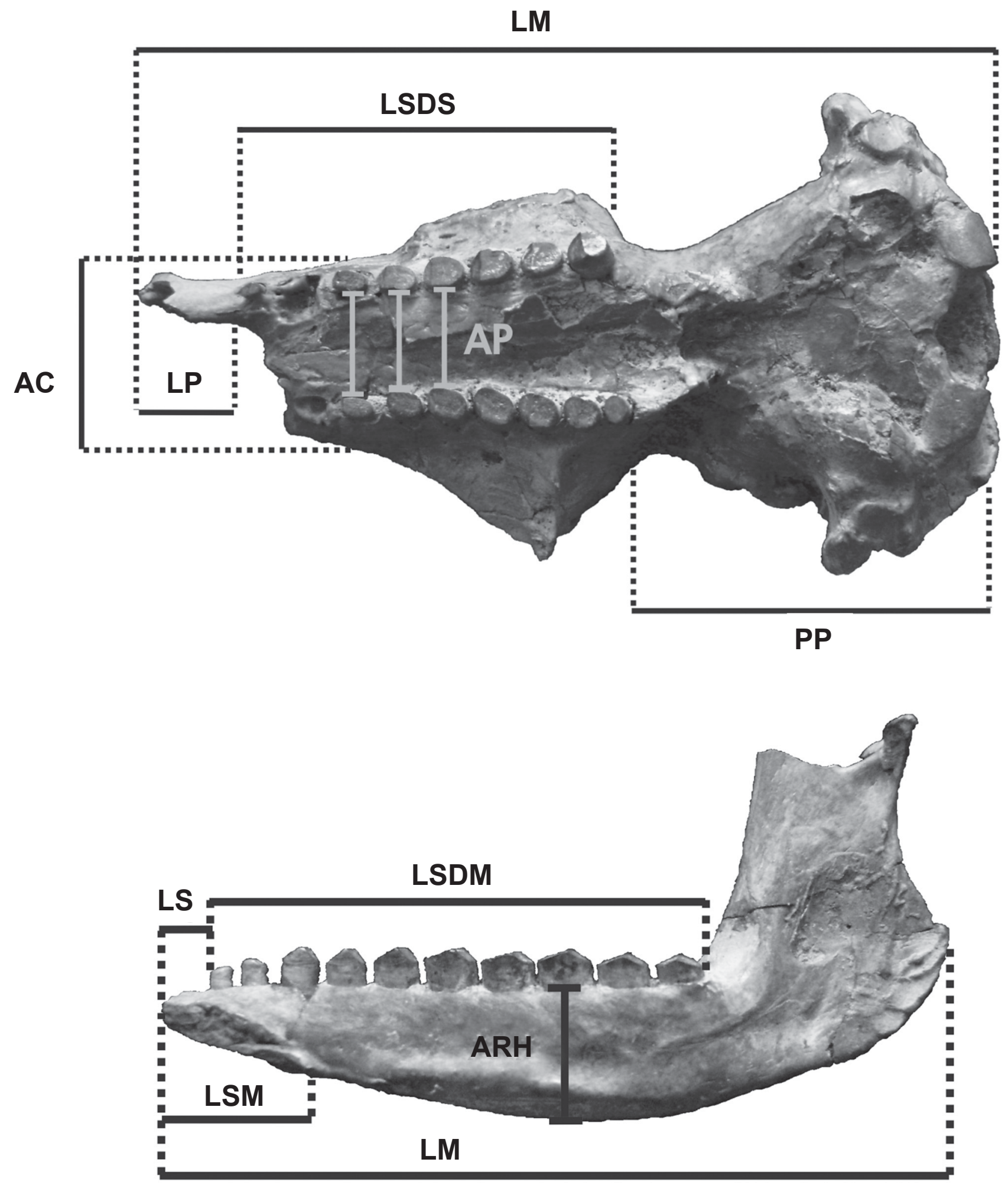

Figura 3. Medidas utilizadas en el trabajo. Ver Material y Métodos para las abreviaturas.

Figure 3. Measurements used in this work. See Material and Methods for abbreviations. 
Todos los valores incluidos en la tabla están expresados en milímetros $(\mathrm{mm})$, con un rango de error de $0,5 \mathrm{~mm}$ y fueron tomados con calibre de mano "Vernier". Se realizó un análisis de componentes principales con los datos morfométricos (Jolliffe, 2002). El cálculo de los autovalores y autovectores se realizó mediante descomposición de valores singulares de los datos estandarizados (centrados y escalados). Todos los cálculos se realizaron utilizando R (R Core Team, 2012). Se comparó además, la disposición del conducto auditivo entre los diferentes ejemplares de las especies consideradas (Carrizo et al., 2005).

La terminología anatómica utilizada proviene principalmente de las contribuciones realizadas por Ameghino (1875), Wible \& Gaudin (2004) y Squarcia et al. (2006). La sistemática se basa en el arreglo propuesto por McKenna \& Bell (1997). El esquema cronológico y bioestratigráfico utilizado en este trabajo corresponde al de Cione \& Tonni (2001, 2005) y Soibelzon et al. (2008).

Abreviaturas institucionales. AAC, Colección Alfredo A. Carlini del Museo de La Plata, La Plata, Argentina; MACN, Museo Argentino de Ciencias Naturales "Bernardino Rivadavia", Buenos Aires, Argentina; MLP, Museo de la Plata, La Plata, Argentina; MUT, Museo de la Universidad de Tarija, Tarija, Bolivia; YPM PU, Yale Peabody Museum of Princeton University, New Haven, Estados Unidos.

Otras abreviaturas. $\mathbf{M}$, molariforme superior; $\mathbf{m}$, molariforme inferior; msnm, metros sobre el nivel del mar.

\section{PALEONTOLOGÍA SISTEMÁTICA}

\author{
Superorden XENARTHRA Cope, 1889 \\ Orden CINGULATA Illiger, 1811 \\ Família DASYPODIDAE Gray, 1821 \\ Tribu EUPHRACTINI Winge, 1923
}

\section{Chaetophractus Fitzinger, 1871}

Especie tipo. Loricatus villosus Desmarest, 1804.

1866 Dasypus (Euphractus) villosus fossilis Burmeister, p. 232.

1880 Euphractus aff. villosus Gervais \& Ameghino, p. 126. 1889 Dasypus aff. villosus Ameghino, p. 865.

1889 Dasypus platensis Ameghino, p. 866.

1902 Dasypus tarijensis Ameghino, p. 257 = Chaetophractus tarijensis (Ameghino 1902).

\section{Chaetophractus villosus (Gray, 1865)}

(Figura 2)

Holotipo. MACN 1612, cráneo parcialmente completo, con la porción dorsal reconstruida en gran parte, sin M1 y M2 derechos ni M2 y M9 izquierdos; hemimandíbula derecha sin el proceso coronoideo y sin la porción más distal de la sínfisis; fragmentos reconstruidos de la hemimandíbula izquierda (Figuras 2A-D).

Procedencia geográfica y estratigráfica. Valle de Tarija, Bolivia. Formación Tolomosa (Pleistoceno).
Material referido. YPM PU 16612, cráneo parcialmente completo, sin ambos arcos cigomáticos y porciones incompletas en frontales y parietales (Figuras 2E-F).

Procedencia geográfica y estratigráfica. Valle de Tarija, Bolivia. Formación Tolomosa (Pleistoceno).

Comentarios. Esta especie fue nombrada por Ameghino (1902) como Dasypus tarijensis, y luego incluida por Hoffstetter (1963) en el género Chaetophractus como C. tarijensis. Takai et al. (1982) figuran y asignan a C. tarijensis una coraza dorsal en buen estado de conservación (MUT-128). Sin embargo, como señalamos anteriormente, esta especie fue fundada en base a material craneano (MACN 1612), con lo cual su asignación específica quedará pendiente para futuros estudios. En trabajos siguientes (Marshall \& Sempere, 1991). Sobre la paleofauna de Tarija se resalta la condición de este taxón como especie endémica; sin embargo, Tonni et al. (2009) indican la necesidad de realizar estudios sistemáticos particulares sobre estos supuestos taxones endémicos. Por último, es importante mencionar que C. villosus fue citado con dudas por algunos autores para el Pleistoceno del Valle de Tarija (Boule \& Thevenin, 1920; Hoffstetter, 1963) en base a de osteodermos aislados.

\section{RESULTADOS Y DISCUSIÓN}

En su descripción original Ameghino (1902) establece comparaciones entre Dasypus tarijensis (= Chaetophractus tarijensis), C. villosus y Euphractus sexcinctus. Indica que $C$. tarijensis es similar en tamaño a Dasypus villosus (= Chaetophractus villosus), pero se distingue de ésta por una serie de caracteres (Ameghino 1902, p. 257, ser. 3a). A continuación se listan y discuten los seis caracteres enunciados por Ameghino (1902, p. 257):

(i) "La parte palatina del intermaxilar que se extiende delante de la primera muela es más largo que en Dasypus villosus, y sucede otro tanto con la parte sinfisaria correspondiente de la mandíbula que es igualmente más larga y más alta". Como se observa en la tabla, estas medidas, en ambos especímenes de Chaetophractus tarijensis, están dentro del rango de las de C. villosus;

(ii) "La región interdentaria del paladar en su parte anterior es más ancha que en Dasypus villosus." En este punto, Ameghino se refiere a la región interdentaria del paladar a nivel del M3 (ver tabla de dimensiones en Ameghino, 1902, p. 257), la cual en el holotipo (MACN 1612) mide 11,33 mm. Este valor es superior en $1 \mathrm{~mm}$ a la mayor medida equivalente tomada en los cráneos de Chaetophractus villosus. En el ejemplar YPM PU-16612 esta medida cae dentro del rango de C. villosus (ver Tabla 1). Esta diferencia podría deberse a que el ejemplar MACN 1612 presenta una fractura en esa zona. Adicionalmente, la región interdentaria entre el M4 y el M5 cae dentro del rango de tamaño de C. villosus;

(iii) "La bóveda palatina se extiende atrás de la última muela, más que en Dasypus villosus y Euphractus sexcinctus." La porción más posterior de la bóveda palatina se encuentra reconstruida en el holotipo. A modo de comprobar si existe diferencia de tamaño en la región postdentaria del cráneo, se 
tomó la distancia máxima entre la parte posterior del M9 y la porción posterior de los cóndilos occipitales (ver Figura 4). En el holotipo (MACN 1612) esta medida resulta ser $1 \mathrm{~mm}$ mayor que en Chaetophractus villosus y $1 \mathrm{~mm}$ menor que $E$. sexcinctus. En el ejemplar YPM PU 16612 esta medida cae en el rango de $C$. villosus (ver Tabla 1);

(iv) "Poco desarrollo de la parte angular de la rama ascendente de la mandíbula". Nuestras observaciones no nos permiten establecer diferencias morfológicas en este sector de la mandíbula del ejemplar tipo y aquellas de Chaetophractus actuales (i.e. C. villosus, C.vellerosus y C. nationi) ni con Euphractus sexcinctus (excepto la diferencia de tamaño). Las dimensiones mandibulares del material en cuestión coinciden con las de C. villosus (ver Tabla 1);

(v) "La forma de la última muela superior no es elíptica sino cilíndrica". Sobre este punto, es necesario realizar algunas aclaraciones. El ejemplar tipo (MACN 1612) estudiado por Ameghino (1902) posee sólo el M9 derecho. Con lo cual, si Ameghino hace referencia a la sección de la superficie oclusal del M9, en el ejemplar tipo ésta es más bien subcircular. Coincidentemente, la morfología de la sección del M9 en el material referido de Chaetophractus tarijensis (YPM PU 16612), Euphractus sexcinctus y Chaetophractus villosus es subcircular (ver Figura 4);

(vi) "La primera muela superior está implantada más verticalmente que en Dasypus villosus, pareciéndose en esto $a$ Euphractus sexcinctus, pero es de tamaño bastante menor que en esta última especie." En los ejemplares estudiados la implantación del M1 es variable. Por ejemplo en el material MACN 14317 asignado a E. sexcinctus el M1 se encuentra levemente inclinado en sentido anterior, y en el material MACN 29726 asignado a Chaetophractus vellerosus el M1 está implantado con una ligera inclinación lingual (ver Figura 4).

Se realizaron dos análisis de componentes principales (PCA) utilizando los datos morfológicos. En un primer análisis, se excluyeron todos aquellos ejemplares que carecen de alguna de las variables, dejando de esta manera fuera al ejemplar YPM PU 16612 de Chaetophractus tarijensis. Los resultados de este análisis mostraron que el $92,46 \%$ de la variabilidad total es explicada por el primer componente principal (CP1), mientras que el segundo componente principal (CP2) explica solamente el 2,4\%. Con el fin de incluir a los dos ejemplares (material tipo y material referido) en el análisis, se realizó un segundo PCA sin las variables faltantes en el YPM PU 16612 (e.g. todas las medidas mandibulares). Los resultados de este segundo análisis mostraron que el $93,56 \%$ de la variabilidad total es explicada por el primer componente principal (CP1), mientras que el segundo componente principal (CP2) explica solamente el 2,51\%. En el gráfico se puede observar como ambos ejemplares pertenecientes a la especie $C$. tarijensis se ubican entre los valores de los ejemplares de C. villosus (ver Figura 5).

Tabla 1. Medidas comparativas (mm). Ver Material y Métodos para las abreviaturas.

Table 1. Comparative measurements ( $\mathrm{mm})$. See Material and Methods for abbreviations

\begin{tabular}{|c|c|c|c|c|c|c|}
\hline & $\begin{array}{l}\text { C. tarijensis } \\
\text { MACN } 1612\end{array}$ & $\begin{array}{l}\text { C. tarijensis } \\
\text { YPMPU } 16612\end{array}$ & C. villosus & C. vellerosus & C. nationi & E. sexcinctus \\
\hline \multicolumn{7}{|l|}{ Medidas } \\
\hline \multicolumn{7}{|c|}{ Craneanas } \\
\hline LM & 93 & 85,4 & $83,63-93,8$ & $54,4-68,9$ & 68,86 & $103,66-121,22$ \\
\hline LP & 8,67 & 9,05 & $7,83-10,4$ & $5,74-8,45$ & 6,64 & $10-12,65$ \\
\hline APM-3 & 11,33 & 9,17 & $8,53-10,35$ & $5,56-7,64$ & 6,9 & $10,83-13,65$ \\
\hline APM-4 & 10,75 & 9,89 & $9,16-11,55$ & $6,36-8,3$ & 7,67 & $10,8-14,6$ \\
\hline APM-5 & 10,9 & 10,3 & $9,81-12,31$ & $6,7-8,7$ & 7,92 & $12-16$ \\
\hline LSDS & 41,16 & 39,59 & $37,07-43,12$ & $24,8-31,86$ & 28,38 & $49,5-56,85$ \\
\hline DTD & 4,78 & 5,65 & $4,1-5,7$ & $2,87-4,55$ & 4 & $4,59-6,7$ \\
\hline DTI & 4,46 & N/A & $4,1-5,5$ & $3-4,44$ & 3,95 & $4,7-6,89$ \\
\hline $\mathrm{AC}$ & 22,7 & 18,55 & $21,35-24,66$ & $12,6-17,7$ & 16,95 & $25,42-47,27$ \\
\hline PP & 37,84 & 34,54 & $32,53-36,52$ & $20,6-25,93$ & 23,7 & $38,8-47,27$ \\
\hline \multicolumn{7}{|c|}{ Mandibulares } \\
\hline LSM & 20,82 & N/A & $16,05-19,8$ & $9,75-13,46$ & 10,18 & $20,64-26,55$ \\
\hline LS & 7,36 & N/A & $4,37-7,78$ & $2,6-4,97$ & 3,37 & $5-7,88$ \\
\hline LSDM & 45,68 & N/A & $42,23-47,2$ & $27,1-34,15$ & 30,66 & $49,5-56,85$ \\
\hline ARH & 12 & 10 & $10,17-12,12$ & $6,23-9$ & 7,65 & $11,36-16,35$ \\
\hline ML & 70,8 & N/A & $67,73-76,3$ & $39,3-53,72$ & 48,55 & $86,62-101,5$ \\
\hline
\end{tabular}



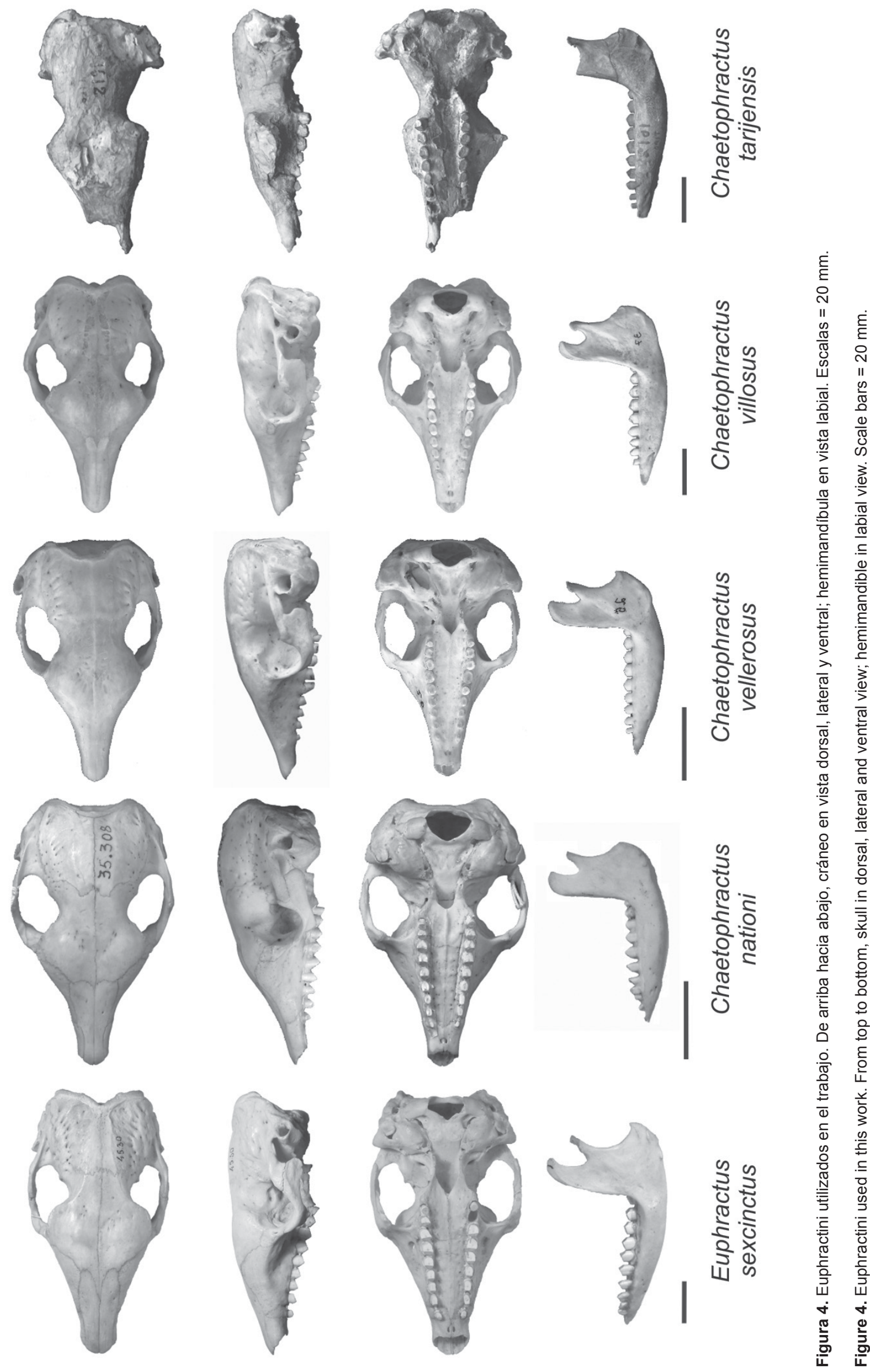


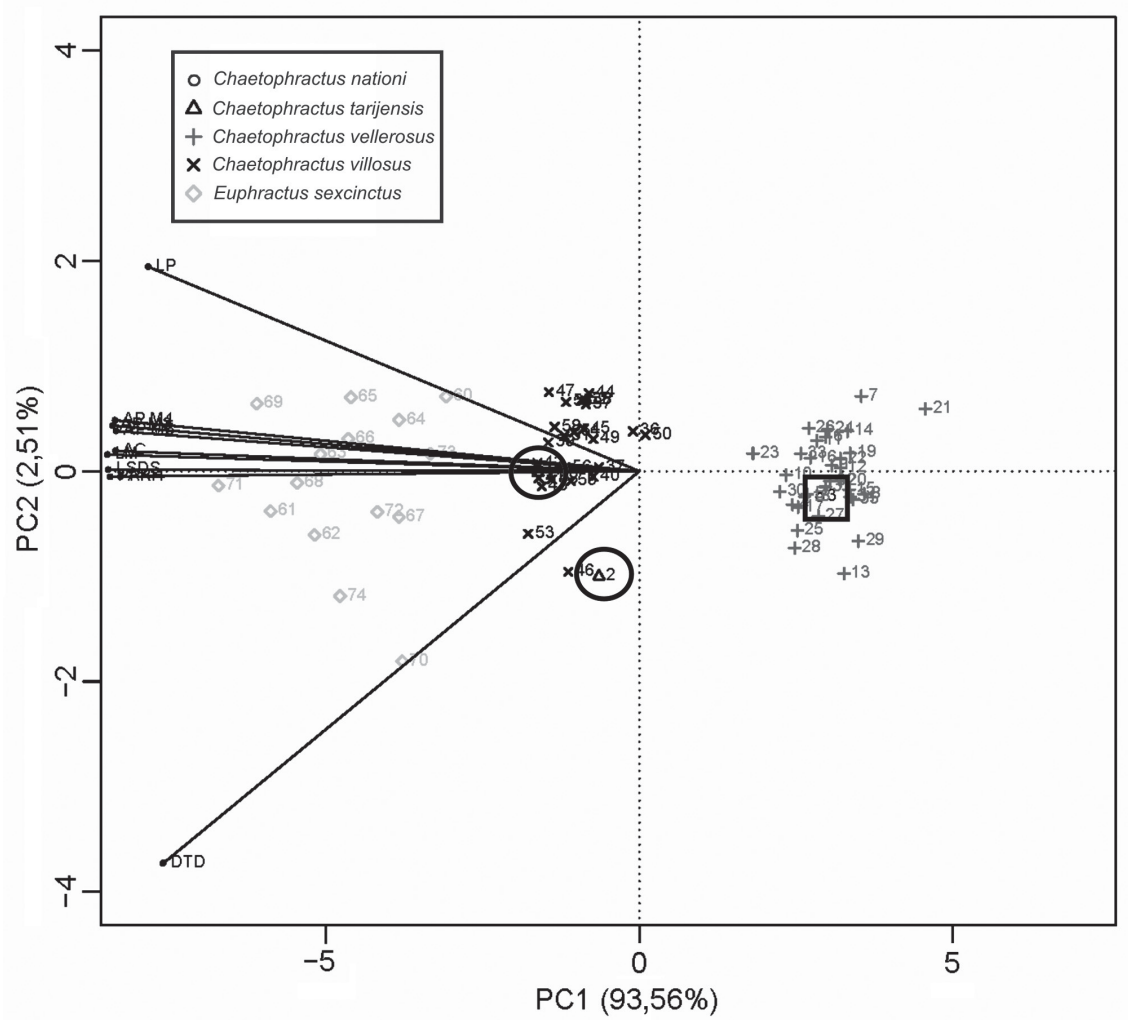

Figura 5. Diagrama mostrando los resultados del Análisis de Componentes Principales (PCA). Los círculos negros indican la ubicación de los ejemplares referidos a Chaetophractus tarijensis y el cuadrado negro la correspondiente a $C$. nationi. Se puede observar que ambos ejemplares pertenecientes a C. tarijensis (MACN 1612 y YMPMU 16612) se ubican dentro del rango de C. villosus. Ver Material y Métodos para las abreviaturas de las medidas utilizadas.

Figure 5. Diagram showing the results of Principal Component Analysis (PCA). The black circles indicate the location of the units related to Chaetophractus tarijensis and the black square correspond to C. nationi. It can be seen that both specimens belonging to $C$. tarijensis (MACN 1612 and YMPMU 16612) are within the range of $C$. villosus. See Material and Methods for abbreviations of the measures used.

Por otro lado, Carrizo et al. (2005) mencionan que las diferencias craneales que permiten diferenciar las especies de Chaetophractus son la disposición del conducto auditivo y el desarrollo de las crestas conformadas por el proceso mastoideo y la osificación del meato auditivo. Así, en $C$. villosus el conducto auditivo es lateral y las crestas están medianamente desarrolladas; en C. vellerosus el conducto es dorsolateral y las crestas están poco desarrolladas, mientras que en $C$. nationi dicho conducto es más dorsal y las crestas mastoideas están bien desarrolladas. Según nuestras observaciones, la morfología de estas estructuras en $C$. tarijensis se condice con aquella descripta por Carrizo et al. (2005) para C. villosus (ver Figura 4). El otro carácter mencionado por Wetzel (1985) para identificar las especies de Chaetophractus (i.e. sutura escamoso-yugal) no es observable en C. tarijensis.

\section{CONCLUSIONES}

El análisis de los datos indica que los caracteres utilizados por Ameghino (1902) para reconocer Dasypus tarijensis (= Chaetophractus tarijensis) (e.g. poco desarrollo de la porción angular de la rama ascendente de la mandíbula, forma de la superficie oclusal del último molariforme) son similares a los observados en C. villosus. Asimismo una comparación detallada del holotipo de $C$. tarijensis con materiales equivalentes de C. villosus, C. vellerosus, C. nationi y Euphractus sexcinctus, junto a un análisis de componentes principales, revelan que este ejemplar (MACN 1612) debe ser asignado a $C$. villosus. Así, de acuerdo con lo establecido en el ICZN (International Commission on Zoological Nomenclature), C. tarijensis pasa a ser sinónimo de C. villosus.

Como ya ha sido mencionado, el Valle de Tarija es uno de los yacimientos pleistocenos más importantes de América del Sur, para el cual numerosos investigadores proponen la presencia de varios taxones endémicos. En revisiones anteriores realizadas por los autores (Soibelzon et al., 2011), se establece que al menos cuatro de las especies mencionadas como endémicas del Valle se registran también en otros yacimientos cuaternarios fuera del Valle de Tarija [i.e. Megatherium tarijensis (Gervais \& Ameghino, 1880), Scelidodon tarijensis, Arctotherium tarijensis, A. wingei Ameghino, 1902]. En este sentido, los resultados de la presente 
contribución aportan valiosa información paleobiogeográfica, ya que confirman la presencia de Chaetophractus villosus en el Pleistoceno del Valle de Tarija. Esto implica una expansión geográfica y temporal de C. villosus, el cual hasta el momento se lo registraba como fósil sólo en Argentina desde el Chapadmalalense (Plioceno medio) a la actualidad (ver Soibelzon et al., 2012), y en Bolivia sólo en tiempos recientes.

\section{AGRADECIMIENTOS}

Los autores de este trabajo desean manifestar su agradecimiento a los curadores de las colecciones paleontológicas visitadas por facilitarnos el acceso a ellas: A. Kramarz, S.M. Alvarez y D. Flores (MACN), M. Reguero (MLP), F.P. Ríos (MUT), D. Brinkman (YPM PU). A.A. Carlini y M. Castro, cuya opinión y discusión para con el trabajo aportó significativamente. A todo el personal del Museo Nacional Paleontológico-Arqueológico de Tarija (F.P. Ríos y L.M. Chamon) por su atención y hospitalidad brindada durante nuestra estadía en la ciudad. Finalmente, a los revisores C. Krmpotic y L. González-Ruiz cuyas correcciones aportaron para mejorar el manuscrito. Este trabajo fue parcialmente financiado por el CONICET, la UNLP y la ANPCyT (PICT 0804, 1664).

\section{REFERENCIAS}

Ameghino, F. 1875. Notas sobre algunos fósiles nuevos de la Formación Pampeana. Buenos Aires, Obras Completas y Correspondencia Científica, p. 1-8.

Ameghino, F. 1902. Notas sobre algunos mamíferos fósiles nuevos o poco conocidos del Valle de Tarija. Anales del Museo Nacional de Buenos Aires, 8:225-261.

Boule, M. \& Thévenin, A. 1920. Mammiféres Fossiles de Tarija. Paris, Imprimerie Nacional, p. 1-256.

Carlini, A.A. \& Scillato-Yané, G.J. 1999. Evolution of Quaternary Xenarthrans (Mammalia) of Argentina. Quaternary of South America and Antarctic Peninsula, 12:149-175.

Carrizo, L.V.; Sánchez M.S.; Mollerach, M.I. \& Barquez, R.M. 2005. Nuevo registro de Chaetophractus nationi (Thomas, 1894) para Argentina; comentarios sobre su identidad sistemática y distribución. Mastozoología Neotropical, 12:233-236.

Ciancio, M.R. \& Carlini, A.A. 2008. Identificación de ejemplares tipo de Dasypodidae (Mammalia, Xenarthra) del Paleógeno de Argentina. Revista del Museo Argentino de Ciencias Naturales, 10:221-237.

Ciancio, M.R.; Carlini, A.A.; Campbell, K.E. \& Scillato-Yané, G.J. 2012. New Palaeogene cingulates (Mammalia, Xenarthra) from Santa Rosa, Perú and their importance in the context of South American faunas. Journal of Systematic Palaeontology, 11:727741. doi: 10.1080/14772019.2012.704949

Cione, A.L. \& Tonni, E.P. 2001. Correlation of Pliocene to Holocene southern South American and European vertebrate-bearing units. Bolletino della Societá Paleontologica Italian, 40:167-173.

Cione, A.L. \& Tonni, E.P. 2005. Bioestratigrafía basada en mamíferos del Cenozoico superior de la provincia de Buenos Aires, Argentina. In: CONGRESO GEOLÓGICO ARGENTINO, 16, 2005. Relatorio, La Plata, p. 183-200.

Coltorti, M.; Abbazzi, L.; Ferreti, M.; Lacumin, P.; Paredes, F.R.; Pellegrini, M.; Pieruccini, P.; Rustioni, M.; Tito, G. \& Rook, L.
2007. Last Glacial mammals in South America: a new scenario from the Tarija Basin (Bolivia). Naturwissenschaften, 94:288299. doi: 10.1007/s00114-006-0196-9

Coltorti, M.; Pieruccini, P. \& Paredes, F.R. 2010. Late Pleistocene stratigraphy, sedimentology and paleoenvironmental evolution of the Tarija-Padcaya basin (Bolivian Andes). Proceedings of the Geologists' Association, 121:162-179. doi: 10.1016/j. pgeola.2010.04.002

Hoffstetter, R. 1963. La faune pléistocéne de Tarija (Bolivie). Bulletin du Muséum National d'Histoire Naturelle, 35:194-203.

Jolliffe, I T. 2002. Principal Component Analysis. $2^{\text {nd }}$ ed. New York, Springer, $487 \mathrm{p}$.

Krmpotic, C.M.; Ciancio, M.R.; Barbeito, C.; Mario, R.C. \& Carlini, A.A. 2009. Osteoderm morphology in recent and fossil Euphractinae xenarthrans. Acta Zoologica, 90:339-351. doi: 10.1111/j.1463-6395.2008.00359.x

MacFadden, B.J; Siles, O.; Zeitler, P.; Johnson, N.M. \& Campbell Jr., K.E. 1983. Magnetic polarity stratigraphy of the middle Pleistocene (Ensenadan) Tarija Formation of southern Bolivia. Quaternary Research, 19:172-187. doi: 10.1016/00335894(83)90003-0

MacFadden, B.J. 2000. Middle Pleistocene climate change recorded in fossil mammal teeth from Tarija, Bolivia, and upper limit of the Ensenadan Land-Mammal Age. Quaternary Research, 54:121-131. doi: 10.1006/qres.2000.2146

MacFadden, B.J. \& Shockey, B.J. 1997. Ancient feeding ecology and niche differentiation of Pleistocene mammalian herbivores from Tarija, Bolivia: morphological and isotopic evidence. Paleobiology, 23:77-100.

MacFadden, B.J.; Zeitler, P.K.; Anaya, F. \& Cottle, J.M. 2013. Middle Pleistocene age of the fossiliferous sedimentary sequence from Tarija, Bolivia. Quaternary Research, 79:268-273. doi: 10.1016/j.yqres.2012.12.009

Marshall, L.G. \& Sempere, T. 1991. The Eocene to Pleistocene vertebrates of Bolivia and their stratigraphic context: a review. Revista Técnica de YPFB, 12:631-652.

McKenna, M.C. \& Bell, S.K. 1997. Classification of mammals above the species level. New York, Columbia University Press, 631 p.

Mones, A. 1986. Paleovertebrata Sudamericana. Catálogo sistemático de los vertebrados fósiles de América del Sur. Parte I. Lista preliminar y bibliografía. Courier Forschungsinstitut Senckenberg, 82:1-625.

Morris, P. 1972. A review of mammalian age determination methods. Mammal Review, 2:69-104. doi: 10.1111/j.1365-2907.1972. tb00160.x

Nowak, R.L. 1999. Walker's Mammals of the World. $6^{\text {th }}$ ed. Baltimore, Johns Hopkins University Press, 1936 p.

$\mathrm{R}$ Core Team (2012). R: a language and environment for statistical computing. R Foundation for Statistical Computing. Available at http://www.R-project.org/; accessed on 07/04/2014.

Rager, L.; Hautier, L.; Forasiepi, A.; Goswami, A. \& SánchezVillagra, M.R. 2013. Timing of cranial suture closure in placental mammals: phylogenetic patterns, intraspecific variation, and comparison with marsupials. Journal of Morphololy, 275:125140. doi: 10.1002/jmor.20203

Scillato-Yané, G.J. 1982. Los Dasypodidae (Mammalia, Edentata) del Plioceno y Pleistoceno de Argentina. Facultad de Ciencias Naturales y Museo. Universidad Nacional de La Plata, Thesis Doctoral, $159 \mathrm{p}$.

Soibelzon, E.; Rodriguez, S.; Avilla L.; Soibelzon, L.H \& Zurita, A.E. 2011. Mammals of the Tarija Valley (Southern Bolivia): diversity, endemism and the problem with its antiquity. In: 
ANNUAL MEETING OF THE SOCIETY OF VERTEBRATE PALEONTOLOGY, 71, 2011. Abstracts, Las Vegas, SVP, p. 197-198.

Soibelzon, E.; Tonni, E.P. \& Bidegain, J.C. 2008. Cronología, magnetoestratigrafía y caracterización bioestratigráfica del Ensenadense (Pleistoceno inferior-medio) en la ciudad de Buenos Aires. Revista de la Asociación Geológica Argentina, 63:421-429.

Soibelzon, E.; Medina, M. \& Abba, A.M. 2012. Late Holocene armadillos (Mammalia, Dasypodidae) of the Sierras of Córdoba, Argentina: zooarchaeology, diagnostic characters and their paleozoological relevance. Quaternary International, 299:72-79. doi: 10.1016/j.quaint.2012.09.009

Squarcia, S.M.; Sidorkewicj, N.S. \& Casanave, E.B. 2006. Cranial osteology of the armadillo Chaetophractus villosus (Mammalia, Xenarthra, Dasypodidae). International Journal of Morphology, 24:541-554. doi: 10.4067/s0717-95022006000500004

Takai, F.; Mizuno, T.; Iwasaki, Y.; Tanaka, K. \& Yoshida, A. 1982. Tarija mammal-bearing formation in Bolivia. Research Institute of Evolutionary Biology, 3:1-72.
Toni, E.P.; Soibelzon, E.; Cione, A.L.; Carlini, A.A.; Scillato-Yané G.J.; Zurita, A.E. \& Paredes, F.R. 2009. Preliminar correlation of the Pleistocene sequences of the Tarija valley (Bolivia) with the Pampean chronological standard. Quaternary International, 210:57-65. doi: 10.1016/j.quaint.2009.06.015

Wetzel, RM. 1985. Taxonomy and distribution of armadillos, Dasypodidae. In: G.G. Montgomery (ed.) The evolution and ecology of armadillos, sloths and vermilinguas, Smithsonian Institution Press, p. 23-46.

Wetzel, R.M.; Gardner, A.L.; Redford, K.H. \& and Eisenberg, J.F. 2007. Order Cingulata. In: A.L. Gardner (ed.) Mammals of South America: Marsupials, Xenarthrans, Shrews and Bats, The University of Chicago Press, p. 128-156.

Wible, J.R. \& T.J. Gaudin. 2004. On the cranial osteology of the yellow armadillo Euphractus sexcinctus (Dasypodidae, Xenarthra, Placentalia). Annals of Carnegie Museum, 73:117196.

Received in May, 2013; accepted in March, 2014.

\section{Apéndice 1/ Appendix 1.}

- Chaetophractus tarijensis: MACN 1612; YPM PU 16612

- Chaetophractus nationi: MACN 35308

- Chaetophractus vellerosus: MACN 2926; 30160; 38252; 48361; 49138; 47378; 30396; 48359; 23667; 34655; 5039; 39410; 3755; 14821; 29726; 48358; 48360; 3278; 48357; 35337; 48366; 14841; 2927; 38254; 312; 24604; 5044. MLP1881. AAC165; 25; $26 ; 175$.

- Chaetophractus villosus: MLP1974; 1991; 1986; 739; 30.V.97.6; 3.X.94.4; 27.X.50.2; 3.X.94.2; 17.V.02.9. AAC176; 45; 49; 36; 176; 177; 178; $179 ; 180 ; 39 ; 41 ; 181 ; 182 ; 19 ; 21 ; 37$.

- Euphractus sexcinctus: MACN23912; 24723; 24743; 50121; 34714; 203; 14317; 4528; 34592; 1124; 4530; 42104; $239 ; 299 ; 4531 ; 204$. 